\title{
JASON 1 : LESSONS LEARNED FROM THE DEVELOPMENT AND 1,5 YEAR IN ORBIT
}

\author{
Thierry.LAFON, \\ JASON 1 Satellite Manager, \\ Centre National d'Etudes Spatiales \\ 18 avenue Edouard Belin - 31401 Toulouse cedex 4 - France \\ thierry.lafon@cnes.fr
}

\section{Abstract}

The JASON-1 small satellite was launched in December 2001 and has performed successfully since one year and a half, with in-flight performances exceeding expectations.

The JASON-1 mission is dedicated to ocean and climate forecasting, in continuation to the successful TOPEXPOSEIDON satellite, launched in 1992.

This project is a cooperation between NASA and CNES. JASON-1 is the first satellite based on the small PROTEUS bus, a cooperative partnership between CNES and Alcatel Space Industries. This project was initiated in 1993 and entered in 1996 in its development phase. At that time, the "Faster, Better, Cheaper" guidelines were driving the decisions and thus strong budgetary and schedule constraints were placed on the project while keeping ambitious technical performances.

After more than one year of Jason operations in orbit, this paper gives a synthetic view of the major project achievement with respect to the initial requirements with a focus on advantages and drawbacks of a fast- paced development and qualification, with emphasis on what has been successful and what would need to be adjusted in a future similar development.

\section{Introduction}

The development of the Jason system was a real challenge for many reasons, among them, one can notice:

- The development of a complete system with a new satellite bus as well as new end users product definition and new control and mission ground segment.

- The continuation of a fruitful NASA/CNES cooperation but with an almost totally reverse responsibility sharing with respect to TOPEX/ POSEIDON organization.

- The first launch of a CNES Satellite on a Delta II launcher from the Vandenberg launch site, in a dual launch configuration.

- The establishment of new relationship between CNES and the Space Industry through partnership instead of standard contractual relations.

- The first CNES try to apply a "Faster, Better, Cheaper" approach.

- The first CNES satellite with routine operations delegated to an other entity.

The excellent behavior of the entire system since launch clearly shows that these challenges have been met with success.

One of the major achievements was to reduce the cost of the mission from about one half, with respect to the previous TOPEX mission, while keeping the same in flight-performances, in less than 5 years of development cycle.

\section{The Jason-1 mission and satellite}

The main scientific objectives for the new Jason-1 altimetry mission were defined after examining feedback from the TOPEX/POSEIDON mission.

The first discipline to benefit from these remarkably accurate tools is physical oceanography. This includes ocean circulation, multi-season to multi-year variability, mesoscale variability, changes in mean sea level, tides and sea state conditions.

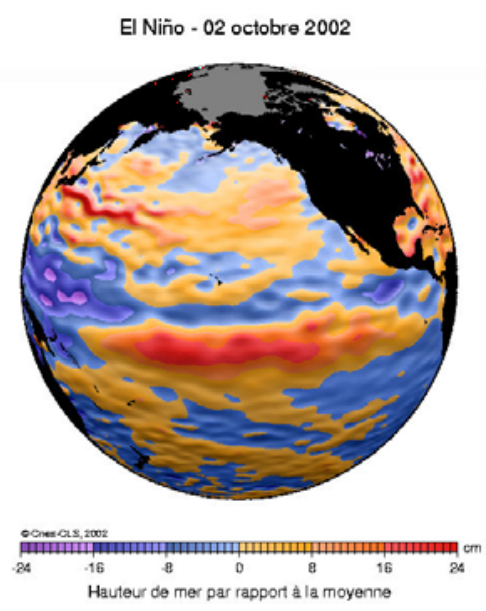

Fig.1. El Nino forecasting

The Jason-1 three axis stabilized satellite is approximately $3.4 \mathrm{~m}$ high and has a total mass of 490 $\mathrm{Kg}(275 \mathrm{Kg}$ for the platform and $175 \mathrm{Kg}$ for the 
payload module including science instruments). Jason1 is about five times lighter than TOPEX.

Once in orbit, the two sets of solar panel of $9.5 \mathrm{~m} 2$ generates about 500 Watts. Jason-1 is designed to last for an operational mission of 3 years and an extended mission of 2 years, all life limited elements being sized for at least 5 years.

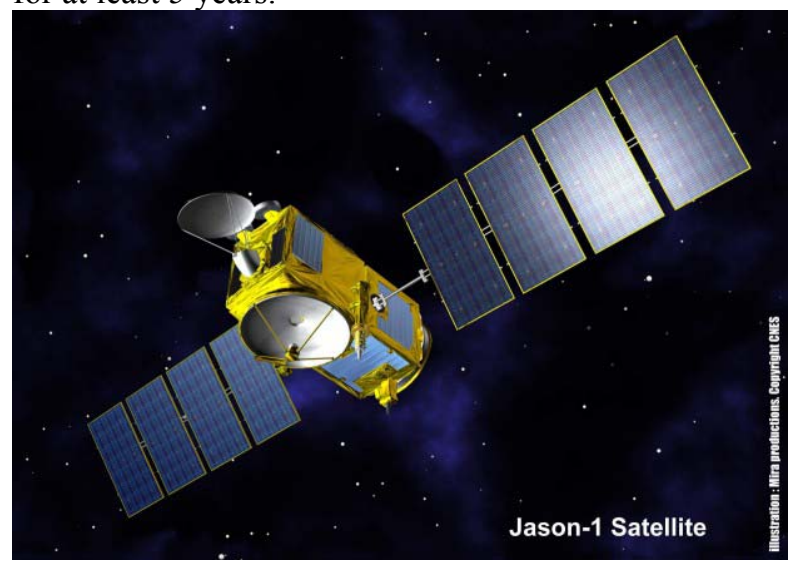

Fig.2 . The JASON-1 Satellite

The satellite is carrying a payload of 5 instruments. A two-frequency altimeter called Poseidon-2 and a DORIS orbitography system are provided by CNES. NASA has provided a three frequency radiometer, a GPS location system and a laser reflector. All these instruments, with the exception of the laser are redundant.

Jason-1 has been launched together with TIMED from the Vandenberg Air Force Base (VAFB) on a DELTA II launch vehicle (7920-10) built by Boeing and provided by NASA, in a Dual Payload configuration with a new Payload Attach Fitting that allow to carry two satellites at the same time.

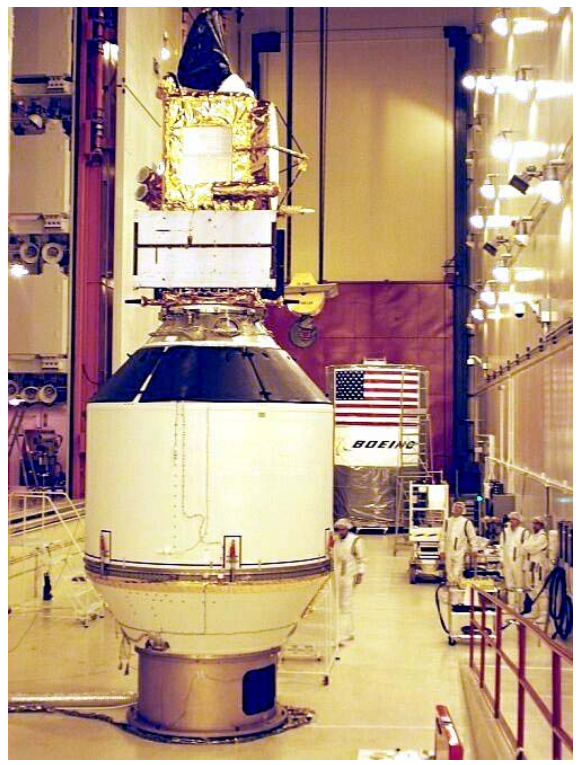

Fig. 3. Jason-1 Launch configuration

\section{The Jason-1 Control ground segment :}

It is comprised of:

- A Jason Command Control Center (JCCC) located in Toulouse that monitors the satellite during the whole mission lifetime.

- A Project Operation Control Center (POCC) located in Pasadena that takes over the routine activities from JCCC at the end of the assessment phase.

- An Earth Terminal Network of three ground stations, located in Aussaguel (France), Poker Flat (USA) and Wallops (USA) as back up site.

\section{The Mission ground segment}

With the prospect of two major altimetry missions, Jason-1 and Envisat (ESA), CNES has implemented a new generation of ground control and science data processing center SSALTO (Segment Sol multi mission d'ALTimetrie, Orbitographie et localisation précise), located at Toulouse.

\section{Jason-1 development context : requirements and constraints}

Jason-1 was the first satellite based on the PROTEUS bus. It was a very ambitious challenge both on a programmatic and technical standpoint to develop and validate a product line and a first satellite in parallel.

From the programmatic standpoint, it was the first partnership which CNES established with a satellite industrial contractor with equal financial participation from both

When, in mid- 1994, following NASA satellite funding shortcut, CNES indicated that the PROTEUS initiative could be the adequate solution to substantially reduce both spacecraft and mission costs, the PROTEUS program had just started Phase A. NASA agreed on this new sharing of responsabilities but the CNES commitment already put the PROTEUS development schedule on the critical path of the project.

PROTEUS was based on flight-proven architectures and technologies, with emphasis on cost efficient procurement and development practices.

The main characteristics of the PROTEUS Platform are given hereafter. The PROTEUS platform will be flown on multiple missions such as the CALIPSO project (NASA/ CNES), the COROT Astronomy satellite and the SMOS (ESA/ CNES) satellite. 


\begin{tabular}{|c|c|}
\hline Orbit & $\begin{array}{l}\text { Any orbit altitude in } 500-1500 \mathrm{Km} \text { range } \\
\text { Orbit inclination higher than } 20 \mathrm{deg}\end{array}$ \\
\hline Launch Vehicles & $\begin{array}{c}\text { Compatible with all launch vehicle with } \\
\text { fairing diameter }>1.9 \mathrm{~m}\end{array}$ \\
\hline Mass & $\begin{array}{c}\text { Bus dry mass }=275 \mathrm{Kg} \\
28 \mathrm{Kg} \text { hydrazine capacity } \\
\text { Payload mass }=100 \text { to } 275 \mathrm{Kg}\end{array}$ \\
\hline Reliability & $\begin{array}{l}0.889 \text { at } 3 \text { years } \\
0.754 \text { at } 5 \text { years }\end{array}$ \\
\hline Lifetime & 3 to 5 years depending upon the orbit \\
\hline Power & $\begin{array}{l}\text { Bus maximum consumption }=300 \mathrm{~W} \\
\text { Payload consumption class }=200 \mathrm{~W} \\
\text { Up to } 300 \mathrm{~W} \text { on some orbits }\end{array}$ \\
\hline $\begin{array}{c}\text { Pointing Attitude } \\
\text { Restitution }\end{array}$ & $0.05 \operatorname{deg}(3$ sigma $)$ on each axis \\
\hline Data Storage & 2 Gbits for payload \\
\hline Down link & $722 \mathrm{kbits} / \mathrm{s}$ \\
\hline Up link & $4 \mathrm{kbits} / \mathrm{sec}$ \\
\hline Unavailability & $0.82 \%$ \\
\hline
\end{tabular}

Table 1. PROTEUS Platform Performances

By the time the development started, the "Faster, Better, Cheaper " paradigm was very common among the small satellites community. This generally translated into several "guidelines" like :

- Use as much as possible electronic parts of "commercial" standard as opposed to usual "space" or "military" standard.

- Strongly "success oriented" schedule with important development and validation phase overlapping and parallel activities.

- Strong reliance on benches to validate the system and its operational behavior.

- Reduction of the number of system and element reviews as well as simplification and alleviation of their progress and minimization of the documentation.

- Maximum use of COT's (Commercial Off The shelf) equipments.

- Decrease of the number of redundancies.

- Limitation in the funding, both applied to development and operational cost.

\section{$\underline{\text { Major Project achievements }}$}

Use as much as possible electronic parts of "commercial" or "military" standard as opposed to usual "space" standard:

Finally, only a very few number of commercial components are actually flying on board Jason-1:

- On the payload instrument side, all the instruments on board Jason-1 had a very strong heritage from the ones flying on TOPEX and it was always argued by the industrial contractor that moving to commercial part will strongly affects this heritage and thus strongly increase overall cost even if the EEE components cost was reduced.

- On the satellite bus, using "off the shelf" equipment was strongly encouraged and those equipment were existing with space or military Thierry LAFON

CNES standard EEE components. Finally, our experience in the use of commercial parts was at that time very limited, especially in a harsh radiation environment like the one existing on the Jason orbit.

In addition, specific procurement rules were to be developed for those new components. At the same time, due to the pre-operational constraint, life time, reliability and availability objectives were not relaxed, and this resulted in a very "classical" approach leading to the use of "known" space or military EEE components.

\section{Strongly "success oriented" schedule with important development phase overlapping and parallel activities:}

To summarize, with respect to the initial planned launch date (March 1999), the total schedule slip was about 19 months of which 6 are directly due to a structural SADM ( Solar Array Drive Mechanism) failure that was discovered very lately in the satellite qualification program, and other 13 month mostly related to test benches development and validation delays which impacted the overall system and operational qualification.

Equipment and instrument delivery and Spacecraft Assembly, Integration and Test process were already close to the critical path. This ambitious schedule was imposed "a priori" without having checked that the relevant resources were available and if the associated "function-sliced" architecture was adequate.

This schedule was an "incentive tool" which proved to be efficient at first but this efficiency strongly decreased when it turned out that the schedule was no longer realistic. The schedule pressure can lead to contradictory objectives. The good compromise is to maintain some "pressure" while keeping reasonable objectives.

In the end, 5 years to design, develop, and validate a complete space system from flight hardware to end users interface, while qualifying a fully new satellite bus, with highly ambitious performances objectives and without accepting strong mission risk increase, remains a remarkable achievement both by the space agencies and the industrial community.

\section{Strong reliance on benches to validate the system and its operational behavior:}

In order to cope with the parallelization of activities, an important part of the validation would have to rely on the satellite simulator bench while running in parallel the satellite Integration and Test activities. But an underestimate of the workload to develop the benches associated with some technical problems related to 
their complexity made the development and validation of the benches themselves much more longer and difficult than expected.

These benches, used for satellite functional validation and for overall system operational qualification, proved to be very efficient with a very good representativity of the real hardware. Thanks to these benches, the test coverage of the Jason system by the time of the launch was very good. For future application, the same strategy will be applied and a numerical simulator, easier to develop and duplicate will be used in addition for the preparation of operations.

\section{Reduction of the number of system and element reviews as well as simplification and alleviation of their progress:}

Phase A and PDR system reviews were "lightened" thanks to a strong system preliminary definition strongly inherited from TOPEX.

For all major Jason satellite or system reviews, the process of the reviews was adapted to fit within 3 working days. An other factor that influenced strongly the review process was the decision taken by NASA to systematically implement a Red Team review process before authorizing a mission launch.

This process was also applied on Jason, from the satellite qualification review until launch. In order to avoid adding more reviews to the process, it was decided to have the NASA Red Team review board participating to the major project reviews in parallel to the CNES Standing Review Board. This process proved to be very efficient with both review groups working closely, each one issuing its own report after coherency checking. In the end, the overall Jason system review process proved to be as efficient as for any other complex project.

\section{Maximum use of COTS (Commercial Off the Shell) equipment:}

Most of the PROTEUS platform equipment were not exactly COTS but derived from existing equipment already used in space application. Their procurement rules, both in terms of contractual rules and technical aspects, have been customized according to their "recurrence" level. Four categories have been defined :

- A) Equipment specifically developed for PROTEUS,

- B) Equipment already developed and qualified for other programs but with modifications needed for PROTEUS,

- C) Equipment already qualified, no modifications required, but a delta qualification (e.g mechanical environment) had to be performed

- D) Equipment already qualified with specifications fully compliant with PROTEUS
This strategy allowed the prime contractor to customize contractual and technical follow-up to each category of equipment and it proved to be a good practice with one exception, the SADM.

This piece of equipment was not considered critical but the combination of organization problems among subcontractors, heavy budgetary constraints, very limited risk assessment and also some "bad luck" circumstances, lead to a very critical situation with the late discovery of a structural failure.

For the star tracker, the platform GPS and also the solar generator, the development was not really straightforward but in each case, Alcatel and CNES set in place the required resources to closely monitor and drive the corrective actions.

\section{Decrease of the number of redundancies:}

This was quickly discarded in view of the very strong impact on the overall reliability. In addition, it was always anticipated to have redundant payload instruments which in case of single string bus makes the satellite reliability figure totally unbalanced between payload and bus. Moreover, it was possible to implement redundancy while staying in the overall total mass cap of $500 \mathrm{Kg}$ with a somewhat marginal impact on the overall cost due to the product line cost decrease effect.

\section{Other lessons learned : \\ $\underline{\text { AI\&T }}$}

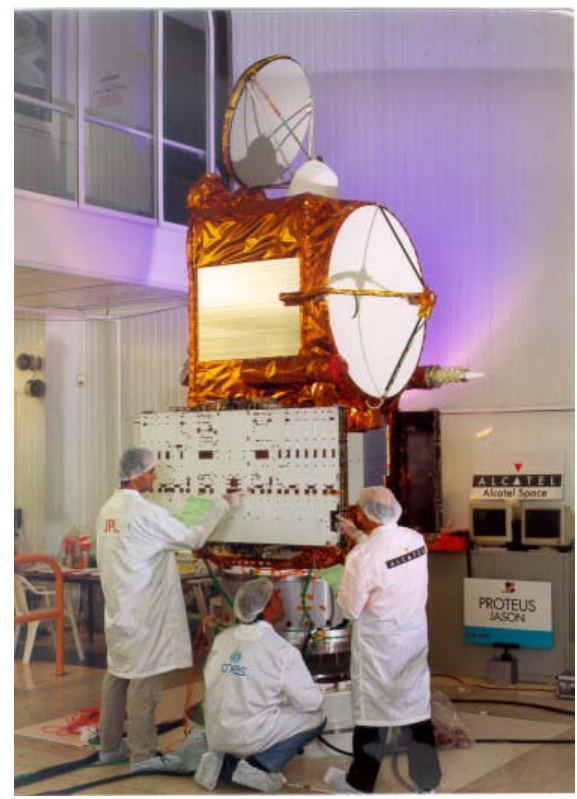

Fig.4. JASON-1 Mechanical test preparation

The AI\&T sequence of activities was sometimes impacted by anomalies which in the end did not impact the schedule due to the other critical path (benches). 
Among the lessons learned, we found out that:

- The Ground Support Equipment used in this sequence shall be carefully validated in its environment before starting satellite integration.

- One should not postpone at a later stage a test that can be performed earlier in the sequence. This is often done due to schedule pressure but proved to be a source of problems occurring later and much more difficult to be identified and fixed.

- The flexibility required when testing a new scientific payload. This dictates frequent modifications of test procedures which is often in conflicts with an industrial streamlined AI\&T sequence. Here again, a compromise has to be found and the rules shall be agreed in advance by all partners.

- Test at the launch site or on the launch pad shall be minimized. If satellite performance check has been correctly done before shipment, only limited "aliveness" test are needed. More over, the different environments between the satellite integration site and the launch site may lead to unexpected differences in the results that are then very difficult to track and explain.

\section{Preparation of operations}

In such a complex system like Jason, with one control center, one operation center, two mission centers and an earth terminal network, the overall operation preparation and training sequence is a long process requiring dedicated resources like a satellite simulator and is usually taking place at the end so most likely on the schedule critical path. The required resources (satellite simulator, RF suitcase...) as well as the system test requirements and constraints were identified very early in the development. The duration of the sequence, starting when the satellite simulator and most of the ground segment individual elements are validated is about year and can not be significantly reduced without an important increase of risk in the early phase of the mission in case of trouble on the satellite. By the time of Jason-1 launch, both CNES and JPL ground systems were fully qualified and opertors trained thanks to a very extensive set of operational test run, addressing nominal and contingency conditions.

\section{NASA/CNES/Alcatel organization}

The Jason project organization was very complex with partnership between NASA and CNES at mission level, but also between CNES and Alcatel on the platform development. CNES acted also as the satellite prime contractor directly procuring the payload sensors (also through JPL). Ground segment elements were also spread out between NASA and CNES.

The management rules between NASA and CNES were described in the Project Plan, directly applicable from the MOU. Various steering committees were defined between NASA and CNES on one hand and CNES and Alcatel on the other hand and adapted along the development of the program in order to have the right people at the right level and with the appropriate knowledge to take critical technical or programmatic decisions.

This organization was efficient and sufficiently flexible to allow a good management process even in the case of "crisis" which sometimes occured.

\section{In Flight Observations}

The JASON-1 satellite has performed successfully since launch. The In-Flight acceptance review has demonstrated the high quality of the spacecraft performances and of the scientific data.

Only one significant anomaly was discovered several months after launch. A progressive degradation of the Star Tracker assembly sensivity lead progressively to frequent tracking losses which could impact in the end the overall pointing accuracy. Nevertheless, the pointing performance was still inside the mission needs, despite the reduced availability.



The phenomenon seems now to be stabilized and the actual conclusions of the Anomaly Review Board is that the loss of sensitivity is probably due to pollution inside the Star Tracker Assembly. As no further degradation has been observed, the actual availability figures are well within the required performances. A back-up plan has nevertheless been prepared to guarantee the Mission continuation. 